\title{
Interferon-gamma release assay levels and risk of progression to active tuberculosis: a systematic review and dose-response meta- regression analysis
}

Jorge R. Ledesma ${ }^{1}$, Jianing Ma ${ }^{1}$, Peng Zheng ${ }^{1,2}$, Jennifer M. Ross ${ }^{3,4}$, Theo $\operatorname{Vos}^{1,2}$ and Hmwe H. Kyu ${ }^{1,2^{*}}$ (1)

\begin{abstract}
Background: Identifying and treating individuals with high risk of progression from latent tuberculosis infection to active tuberculosis (TB) disease is critical for eliminating the disease. We aimed to conduct a systematic review and meta-regression analysis to quantify the dose-response relationship between interferon-gamma release assay (IGRA) levels and the risk of progression to active TB.

Methods: We searched PubMed and Embase from 1 January 2001 to 10 May 2020 for longitudinal studies that reported the risk of progression from latent to active TB as a function of baseline IGRA values. We used a novel Bayesian meta-regression method to pool effect sizes from included studies and generate a continuous doseresponse risk curve. Our modeling framework enabled us to incorporate random effects across studies, and include data with different IGRA ranges across studies. The quality of included studies were assessed using the NewcastleOttawa scale (NOS).

Results: We included 34 studies representing 581,956 person-years of follow-up with a total of 788 incident cases of TB in the meta-regression analysis. Higher levels of interferon-gamma were associated with increased risk of progression to active tuberculosis. In the dose-response curve, the risk increased sharply between interferon-gamma levels 0 and $5 \mathrm{IU} / \mathrm{ml}$, after which the risk continued to increase moderately but at a slower pace until reaching about $15 \mathrm{IU} / \mathrm{ml}$ where the risk levels off. Compared to $0 \mathrm{IU} / \mathrm{ml}$, the relative risk of progression to active TB among those with interferon-gamma levels of $0.35,1,5,10,15$, and $20 \mathrm{IJ} / \mathrm{ml}$ were: 1.64 (1.28-2.08), 2.90 (2.02-3.88), 11.38 (6.64-16.38), 19.00 (13.08-26.90), 21.82 (14.65-32.57), and 22.31 (15.43-33.00), respectively. The dose-response relationship remains consistent when limiting the analysis to studies that scored highest in the NOS.

Conclusion: The current practice of dichotomizing IGRA test results simplifies the TB infection disease continuum. Evaluating IGRA test results over a continuous scale could enable the identification of individuals at greatest risk of progression to active TB.
\end{abstract}

Keywords: Latent tuberculosis, IGRA, Active tuberculosis, Dose-response meta-regression

\footnotetext{
* Correspondence: hmwekyu@uw.edu

'Institute for Health Metrics and Evaluation, University of Washington, 3980 15th Ave. NE, Seattle, WA 98195, USA

${ }^{2}$ Department of Health Metrics Sciences, University of Washington, 3980 15th Ave. NE, Seattle, WA 98195, USA

Full list of author information is available at the end of the article
}

(C) The Author(s). 2021 Open Access This article is licensed under a Creative Commons Attribution 4.0 International License, which permits use, sharing, adaptation, distribution and reproduction in any medium or format, as long as you give appropriate credit to the original author(s) and the source, provide a link to the Creative Commons licence, and indicate if changes were made. The images or other third party material in this article are included in the article's Creative Commons licence, unless indicated otherwise in a credit line to the material. If material is not included in the article's Creative Commons licence and your intended use is not permitted by statutory regulation or exceeds the permitted use, you will need to obtain permission directly from the copyright holder. To view a copy of this licence, visit http://creativecommons.org/licenses/by/4.0/. The Creative Commons Public Domain Dedication waiver (http://creativecommons.org/publicdomain/zero/1.0/) applies to the data made available in this article, unless otherwise stated in a credit line to the data. 


\section{Background}

Tuberculosis (TB) is the leading cause of mortality from a single infectious agent, with more than 1 million deaths per year [1]. Mycobacterium tuberculosis (Mtb) is the causative agent of TB, though a person with Mtb infection can remain asymptomatic in a state known as latent TB infection (LTBI). While individuals with LTBI are asymptomatic, they are an important reservoir for future TB cases. Approximately one quarter of the world's population is estimated to have LTBI $[2,3]$, of which 5 to $15 \%$ will develop active $\mathrm{TB}$ at some point in their lives [4]. Identifying individuals with LTBI and placing those at risk of developing active TB on preventive treatment is thus critical for eliminating the disease [5].

The tuberculin skin test (TST) has traditionally been used to test for latent TB infection, but has known limitations. Most notably, its specificity is adversely affected by BCG vaccination or infection with nontuberculous mycobacteria. Additionally, TST requires multiple health care visits over $48-72 \mathrm{~h}$ to place the test and read the result, which may be prone to inter-reader variability [6]. Alternatives to the TST are T-cell-based interferongamma release assays (IGRAs). Two forms of IGRAs are currently available for commercial use that are widely used: QuantiFERON TB Gold in tube (QFT-GIT) and T-SPOT.TB. IGRAs have several advantages over TST such as higher specificity for Mtb and lack of cross reaction with BCG vaccination. IGRAs have become the primary diagnostic tool for LTBI in low to intermediate TB burden countries [7]. Despite the higher cost of the test and some challenges with reproducibility, the use of IGRAs is expected to further expand in high TB burden countries, as the World Health Organization (WHO) has recently endorsed the use of QFT-GIT and TSPOT.TB for the End TB strategy [8].

Given the likely increased use of QFT-GIT in high TB burden settings, it is critical to review the utility of QFTGIT to predict progression from latent to active TB. A recent meta-analysis of cohort studies indicated that $\mathrm{TB}$ contacts with a positive IGRA result have a 10.8-fold higher rate of progression to active TB [9]. In addition, recent individual studies have suggested a need to further examine the entire distribution of IGRA values for risk analyses of subsequent active TB [10-12]. These studies have shown a marked increase in the risk of incident active TB disease with higher IGRA values. Some researchers have called for a need to report a borderline zone, an intermediate area between a negative and positive IGRA test, to improve the diagnostic accuracy of potential development of active TB [13-15]. Together the literature suggests that there is potentially a loss of information for identification of individuals at high risk of active TB when IGRA results are dichotomized by the traditional $0.35 \mathrm{IU} / \mathrm{ml}$ threshold.
Previously published systematic reviews and metaanalyses of IGRA performance have yet to take into account the full spectrum of the infection by considering IGRA values at a continuous scale. A consideration of the entire distribution of IGRA levels rather than a binary cut-off may help inform treatment considerations for those at the highest risk of progressing to active TB. We aimed to conduct a systematic review and meta-analysis to quantify the dose-response relationship between IGRA levels and risk of progression to active TB using all available global data sources.

\section{Methods}

\section{Literature search}

In this systematic review and meta-regression analysis we followed the PRISMA and MOOSE checklists. We searched PubMed and Embase from 1 January 2001 to 10 May 2020 for studies that reported the risk of progression from latent to active TB based on baseline IGRA values. The full search strategy is available in the supplementary material. In addition, we made no restrictions in study language. The reference lists of eligible full texts and identified systematic reviews were reviewed for additional relevant studies.

\section{Study selection}

Retrospective or prospective cohort studies and clinical trials that assessed QuantiFERON-TB Gold in tube (QFT-GIT) or QuantiFERON-TB Gold Plus (QFT-Plus) results, defined as the difference of interferon-gamma level between the TB antigen tube and negative control tube, as the exposure variable and progression to active tuberculosis as an outcome, were eligible for inclusion. The study participants were adults or children who were free of active TB disease at baseline. We excluded studies that contained a sample with a confounding disease (e.g. lung cancer, rheumatic diseases, inflammatory bowel diseases), that did not test all individuals with QuantiFERON-TB, or did not follow-up all individuals for progression to active TB disease. We further excluded studies that only focused on QuantiFERON-TB conversion or had participants with previous positive QuantiFERON-TB tests.

\section{Data extraction}

Data were extracted using a standardized data extraction form developed a priori. The following variables were extracted from each study: study design, location, follow-up duration, sample attrition rate, baseline agesex distribution, TB preventive treatment use, sample size by baseline QuantiFERON-TB results (IU/ml categories), number of cases progressing to active TB by $\mathrm{IU} / \mathrm{ml}$ categories, and method for diagnosing active TB. Information on participant characteristics (e.g. general 
population, TB contacts, healthcare workers, etc.) was also extracted. In studies that separately reported data from participants who developed TB very early (under 2 months of the start of the study), they were considered as prevalent TB cases and were excluded during extraction such that they did not contribute to the sample size for baseline QuantiFERON-TB results nor for the number of incident TB cases. When available, extracted data were stratified by TB preventive treatment use. For person-year information, we used the study mean or median as follow-up time if follow-up time was not disaggregated for every QuantiFERON-TB category.

\section{Assessment of quality of included studies}

We examined the quality of included studies using the Newcastle-Ottawa quality assessment scale (NOS) [16]. Potential scores range from 0 to 9 points, with higher scores representing higher quality studies. Study scores were based on the following criteria: selection of the study population, comparability between exposed and non-exposed groups, and assessment of the outcome.

\section{Data analysis}

Incidence rate ratios (referred to as relative risks in this paper) for each study were computed by using the total number of cases that progressed to active TB and accumulated person-time information for each IGRA category. We then used a novel Bayesian meta-regression method [17] to analyze data from included studies and generate a continuous risk curve for the association between IGRA values and risk of progression to active TB. This method allowed us to incorporate random-effects across studies and include heterogeneous data with various IGRA categories. The primary advantage of this method is that it is able to take any IGRA range (e.g. 0$0.35,0.35-1,4-10,0.35-20$, etc.) as input and use integration techniques to derive continuous risks over the entire distribution of IGRA values. Detailed methods and equations can be found in the supplementary material. We separately analyzed studies where the study population were people living with HIV (PLHIV).

\section{Sensitivity analyses and subgroup analyses}

We assessed the robustness of the dose-response risk curve by conducting a sensitivity analysis stratifying results by their NOS score. We assessed for the potential of effect modification by study-level population. The population subgroup analyses included: TB case contacts, healthcare workers, migrants, PLHIV, adults $(\geq 18$ years), and children (<18 years). We conducted additional subgroup analyses by stratifying results by whether studies provided any TB preventive treatment (TPT) or not. Across the subgroup analyses, all model parameters for the Bayesian meta-regression remained consistent with the primary analysis. To investigate differences in the dose-response curves between subgroups we generated 1000 estimates from the posterior distributions to generate a ratio of the relative risks with uncertainty.

\section{Results}

Our literature search identified a total of 1074 citations (Fig. 1). After removal of duplicate citations, 884 remained for title and abstract review of which 82 were included for full-text assessment. We included 34 studies in our dose-response meta-analysis, 18 of which were conducted in Europe, 8 in Asia, 2 in North America, 2 in the Middle East, 2 in Sub-Saharan Africa, 1 in Latin America, and 1 in Australasia. The 34 studies provided a combined cohort size of 581,956 person-years with a total of 788 incident cases of TB. The median study duration was 2.5 years (IQR 1.75-4.30).

Most study cohorts included participants at elevated risk for TB (Table S1). Of the included studies, 15 cohorts included TB case contacts, 8 cohorts of healthcare workers, and 5 cohorts included migrants or asylum seekers. In addition, 2 studies were population-based and another study included individuals without known exposure to TB. Eight studies provided TPT to participants ranging from 2 to $5 \%$ of the study sample with one study providing treatment to $19 \%$ of the study sample, while 16 studies did not provide TPT.

\section{Dose-response relationship}

Overall, higher levels of IFN-gamma were associated with increased risk of progression to active tuberculosis (Fig. 2). We find that the risk increased sharply between IFN-gamma levels 0 and $5 \mathrm{IU} / \mathrm{ml}$, after which the risk continued to increase moderately but at a slower pace until reaching about $15 \mathrm{IU} / \mathrm{ml}$ where the risk levels off until $20 \mathrm{IU} / \mathrm{ml}$. Table 1 provides quantitative measurements of the dose-response risk curve for the primary analysis. Specifically, the relative risk of progression to active TB compared to $0 \mathrm{IU} / \mathrm{ml}$ for those with IFNgamma levels of $0.20,0.35,0.70,1,5,10,15$, and $20 \mathrm{IU} /$ ml were: 1.37 (1.15-1.63), 1.64 (1.28-2.08), 2.31 (1.633.09), 2.90 (2.02-3.88), 11.38 (6.64-16.38), 19.00 (13.0826.90), 21.82 (14.65-32.57), and 22.31 (15.43-33.00), respectively.

\section{Sensitivity analyses}

The quality assessment scores for each study are in Table S1. According to the quality assessment, the quality of the including studies ranged between 6 , indicating moderate quality, and 3 , indicating lower quality. Particularly, $14(41 \%)$ studies scored between 5 and 6,12 (35\%) studies scored a 4, and the remaining $8(24 \%)$ studies scored a 3 . Sensitivity analyses that excluded 

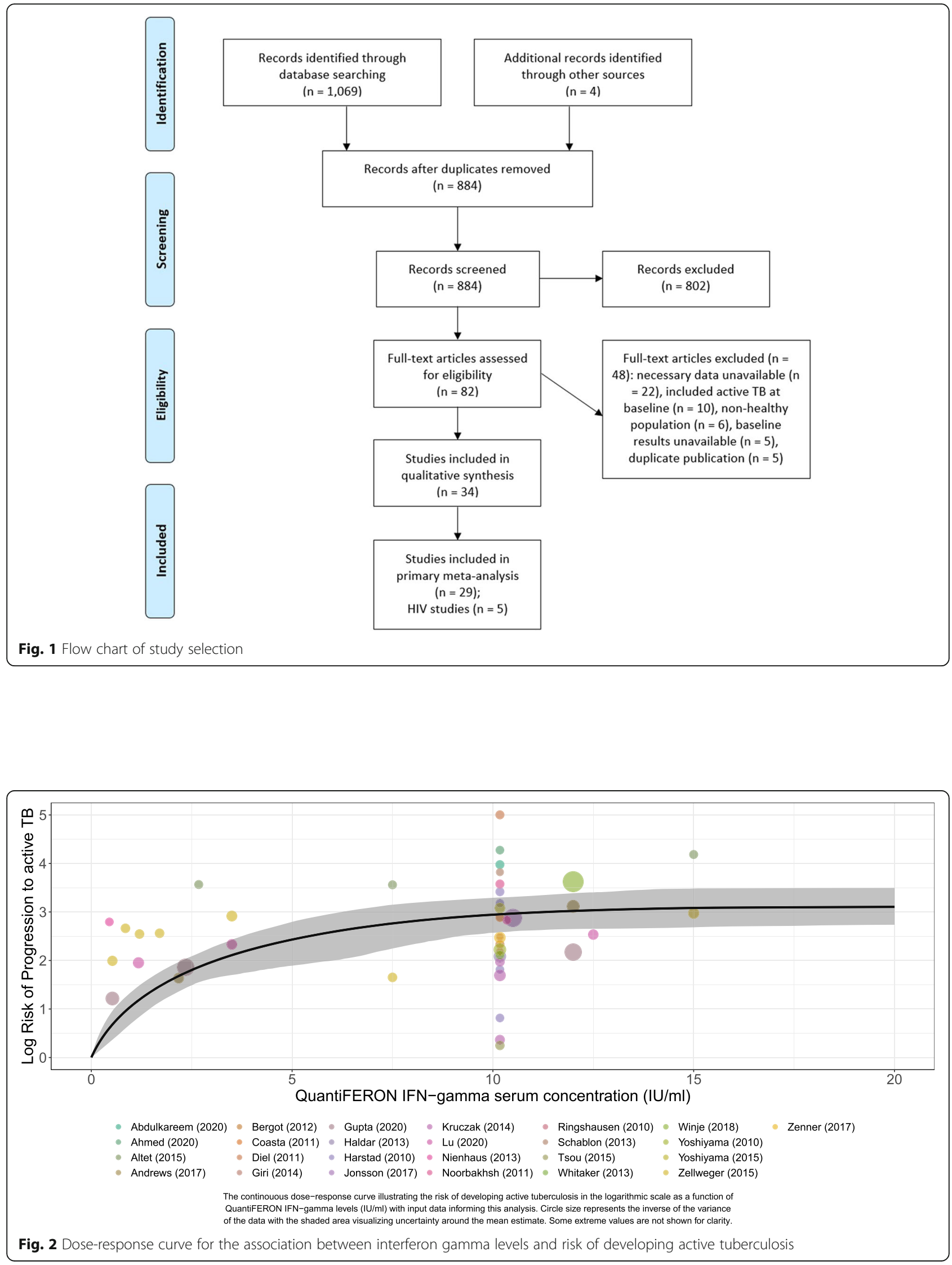
Table 1 Risk of progression to active TB across IFN-gamma levels (IU/ml) by model

\begin{tabular}{|c|c|c|c|c|c|c|}
\hline \multirow{2}{*}{$\begin{array}{l}\text { IFN-gamma } \\
\text { levels (IU/ml) }\end{array}$} & \multicolumn{6}{|c|}{ Relative Risk (95\% Uncertainty interval) } \\
\hline & Primary Analysis & $\begin{array}{l}\text { High Quality } \\
\text { Studies }^{\mathrm{a}}\end{array}$ & Received TPT & No TPT & Adults & Children \\
\hline 0.00 & REF & REF & REF & REF & REF & REF \\
\hline 0.10 & 1.18 (1.07 to 1.32$)$ & $1.23(1.09$ to 1.39$)$ & $1.26(1.05$ to 1.52$)$ & $1.16(1.02$ to 1.32$)$ & $1.24(1.10$ to 1.39$)$ & $1.13(1.00$ to 1.41$)$ \\
\hline 0.20 & $1.37(1.15$ to 1.63$)$ & $1.45(1.19$ to 1.77$)$ & $1.50(1.10$ to 2.01$)$ & $1.32(1.05$ to 1.64$)$ & $1.46(1.21$ to 1.76$)$ & $1.26(1.00$ to 1.81$)$ \\
\hline 0.30 & 1.55 (1.23 to 1.93$)$ & $1.67(1.29$ to 2.14$)$ & $1.74(1.16$ to 2.50$)$ & $1.48(1.10$ to 1.94$)$ & 1.69 (1.32 to 2.12$)$ & 1.37 (1.01 to 2.19) \\
\hline 0.35 & 1.64 (1.28 to 2.08$)$ & 1.78 (1.34 to 2.32$)$ & $1.86(1.19$ to 2.74$)$ & $1.56(1.12$ to 2.09$)$ & $1.80(1.37$ to 2.30$)$ & 1.43 (1.01 to 2.38$)$ \\
\hline 0.40 & $1.74(1.32$ to 2.24$)$ & $1.88(1.39$ to 2.50$)$ & $1.98(1.23$ to 2.97$)$ & 1.64 (1.15 to 2.25$)$ & $1.90(1.42$ to 2.47$)$ & 1.49 (1.01 to 2.57$)$ \\
\hline 0.50 & 1.93 (1.42 to 2.54$)$ & 2.10 (1.50 to 2.84$)$ & 2.22 (1.30 to 3.43$)$ & 1.81 (1.22 to 2.54$)$ & 2.11 (1.53 to 2.81 ) & 1.60 (1.01 to 2.95$)$ \\
\hline 0.70 & 2.31 (1.63 to 3.09 ) & 2.52 (1.71 to 3.53$)$ & 2.68 (1.47 to 4.33$)$ & 2.15 (1.38 to 3.09$)$ & 2.52 (1.75 to 3.45$)$ & 1.81 (1.02 to 3.67) \\
\hline 1.00 & 2.90 (2.02 to 3.88$)$ & 3.14 (2.10 to 4.45$)$ & 3.37 (1.81 to 5.64$)$ & 2.67 (1.68 to 3.93 ) & 3.09 (2.10 to 4.32$)$ & 2.13 (1.03 to 4.73$)$ \\
\hline 2.00 & 4.99 (3.61 to 6.65$)$ & 5.08 (3.29 to 7.50$)$ & 5.59 (3.01 to 9.41$)$ & 4.56 (3.03 to 6.52 ) & 4.70 (3.15 to 6.66$)$ & 3.10 (1.06 to 7.67$)$ \\
\hline 3.00 & 7.16 (5.07 to 9.65$)$ & $6.80(4.27$ to 10.67$)$ & 7.65 (4.01 to 12.38$)$ & 6.54 (4.09 to 9.88 ) & 5.89 (3.93 to 8.25$)$ & 3.95 (1.15 to 11.06$)$ \\
\hline 4.00 & 9.32 (5.85 to 13.06$)$ & 8.31 (4.69 to 14.30 ) & 9.53 (4.75 to 15.19$)$ & 8.50 (4.62 to 13.49$)$ & 6.73 (4.16 to 9.57$)$ & $4.72(1.28$ to 14.53$)$ \\
\hline 5.00 & 11.38 (6.64 to 16.38$)$ & 9.63 (4.87 to 18.00$)$ & 11.26 (5.52 to 18.08$)$ & $10.42(4.85$ to 17.51$)$ & 7.33 (4.31 to 10.81$)$ & 5.48 (1.45 to 18.14$)$ \\
\hline 6.00 & 13.31 (7.37 to 19.43$)$ & 10.83 (5.17 to 21.46) & 12.90 (6.33 to 20.97) & 12.28 (5.31 to 21.43$)$ & 7.80 (4.50 to 12.19$)$ & 6.30 (1.72 to 21.91$)$ \\
\hline 7.00 & 15.07 (8.63 to 22.07) & 11.99 (5.67 to 24.60$)$ & 14.54 (7.61 to 23.07) & 14.11 (6.38 to 24.60$)$ & 8.27 (5.01 to 13.19$)$ & 7.23 (2.03 to 25.37$)$ \\
\hline 8.00 & 16.61 (10.31 to 24.16) & 13.13 (6.29 to 27.22$)$ & 16.17 (9.37 to 25.01) & 15.84 (8.08 to 26.98) & 8.79 (5.62 to 14.14$)$ & 8.25 (2.46 to 27.55$)$ \\
\hline 10.00 & 19.00 (13.08 to 26.90$)$ & 15.16 (7.38 to 31.92) & 19.10 (12.87 to 29.37$)$ & 18.74 (11.01 to 30.36$)$ & 9.85 (6.20 to 15.17$)$ & 10.24 (2.96 to 33.94$)$ \\
\hline 12.00 & 20.53 (14.13 to 29.62) & 16.74 (7.90 to 35.79$)$ & 21.35 (14.11 to 32.55$)$ & 20.75 (12.04 to 34.15$)$ & 10.78 (6.79 to 16.66$)$ & 11.90 (3.47 to 39.68$)$ \\
\hline 15.00 & 21.82 (14.65 to 32.57$)$ & 18.18 (8.39 to 40.54$)$ & 23.26 (14.95 to 37.08$)$ & 22.39 (12.88 to 38.79) & 11.75 (7.14 to 18.99$)$ & 13.44 (4.03 to 45.99$)$ \\
\hline 20.00 & 22.31 (15.43 to 33.00$)$ & 18.74 (8.69 to 41.61$)$ & 23.82 (15.26 to 37.92 ) & 22.94 (13.27 to 39.56$)$ & 12.22 (7.43 to 19.48$)$ & 14.04 (4.33 to 46.61$)$ \\
\hline
\end{tabular}

${ }^{\mathrm{a}}$ Sensitivity analysis excluding studies with NOS score $<5$

NOTE: TPT (TB preventive treatment). In the TPT subgroup analyses, data inputs into the models were stratified by whether studies provided any TPT. The children subgroup analysis included data for individuals below 18 years, while the adult subgroup analysis included data for those 18 years and older

studies with an NOS score lower than 5 exhibit similar results to models utilizing the complete dataset (Fig. 3; Table 1). While the curve including high-quality studies was steeper at the lower end and lower at the higher end compared to the primary analysis, the differences were not statistically significant.

\section{Subgroup analyses}

In subgroup analyses, there was some heterogeneity in risk across IFN-gamma levels for at risk populations. The risk of TB among people living with HIV (PLHIV) was higher compared to the rate found in the primary analysis. Across all levels of IFN-gamma, the doseresponse risk curve for PLHIV was substantially higher compared to the primary analysis (Fig. 4a; Table 2). Among TB case contacts (Fig. 4b; Table 2) and migrants (Fig. 4c; Table 2) the risk was steeper at the lower end of IFN-gamma levels $(<2.5 \mathrm{IU} / \mathrm{ml})$ but the risk was lower in the remaining IFN-gamma levels compared to the primary analysis. For healthcare workers (Fig. 4d; Table 2), the dose-response risk curve yielded similar results to the primary analysis. Similarly, we found no difference in the risk of progression to active $\mathrm{TB}$ when stratifying results by whether studies provided TPT. (Fig. 4e; Table 1 ). Finally, we found that the dose-response risk curve was steeper at the lower end $(<7.5 \mathrm{IU} / \mathrm{ml})$ for adults compared to children but the risks converged afterwards (Fig. 4f; Table 1). Additional subgroup analyses on time to incident $\mathrm{TB}$, definition of active $\mathrm{TB}$, and $\mathrm{TB}$ incidence of country of study showed no significant differences (Fig. S1-S3).

\section{Discussion}

To our knowledge, this is the first meta-analysis to summarize and quantify the dose-response relationship between QuantiFERON-TB IFN-gamma levels and the risk of progression to active TB using all available global data sources. Using data from 34 studies, we found that the risk of TB development increased with higher IFNgamma levels. Our continuous dose-response curve indicates that the risk of incident TB sharply increases between IFN-gamma levels of 0 and $5 \mathrm{IU} / \mathrm{ml}$ after which the risk continued to increase moderately but at a slower pace until reaching $15 \mathrm{IU} / \mathrm{ml}$ where the risk levels off. Sensitivity analyses revealed that our findings are robust 


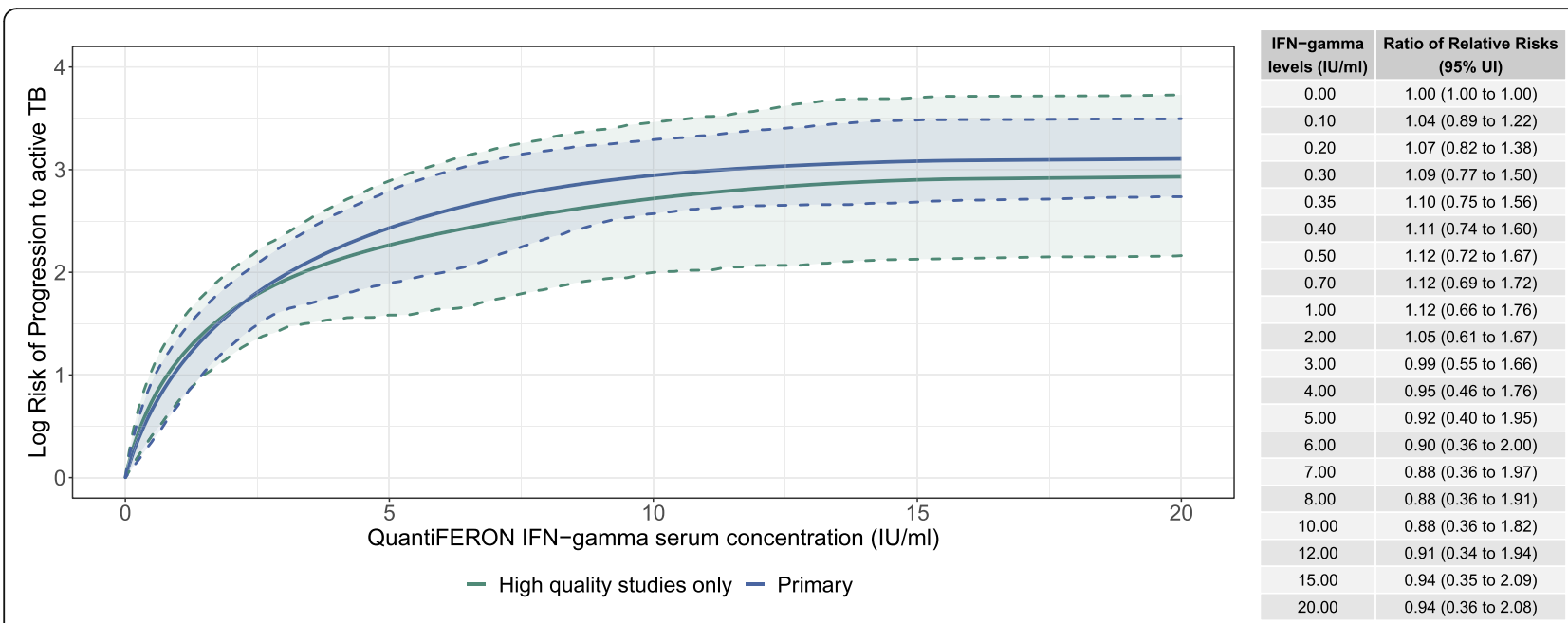

Note: The ratio of relative risks were computed by dividing the relative risks from the model including only high quality studies $(\mathrm{N}=11)$ by those from the Primary model

Fig. 3 Sensitivity analysis results (excluding studies with NOS quality score $<5$ )
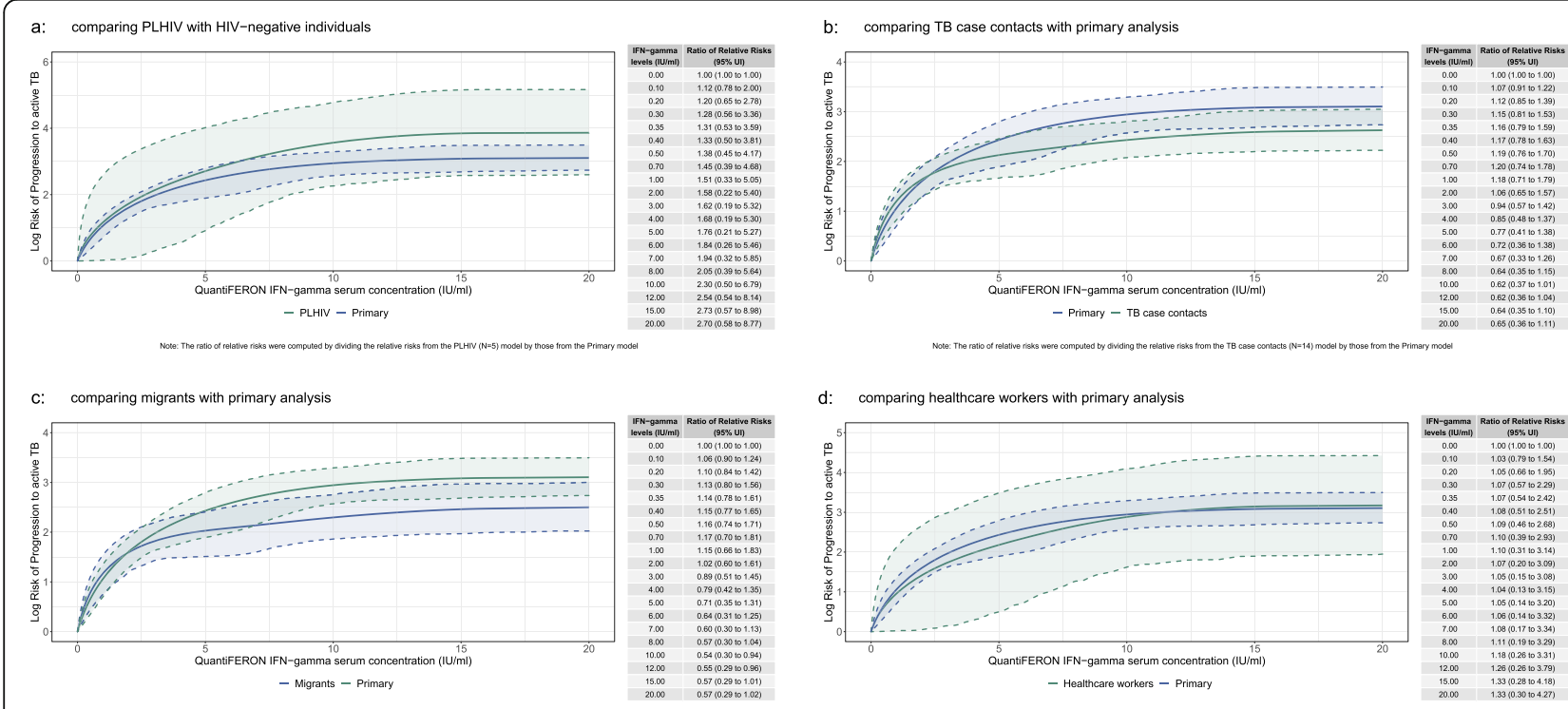

d: comparing healthcare workers with primary analysis
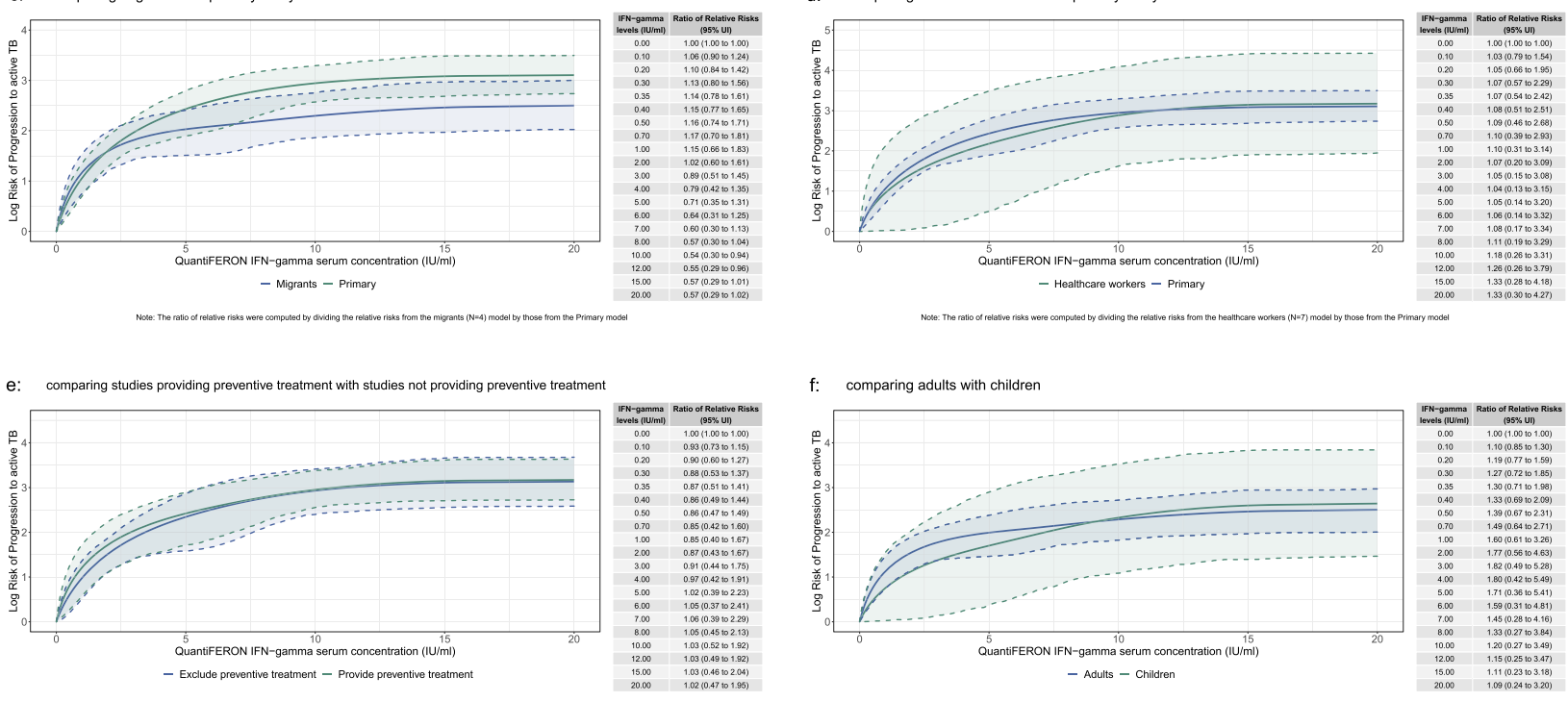

Fig. 4 Subgroup analysis results: a comparing PLHIV with HIV-negative individuals, b comparing TB case contacts with primary analysis, c comparing migrants with primary analysis, $\mathbf{d}$ comparing healthcare workers with primary analysis, e comparing studies providing preventive treatment with studies not providing preventive treatment, $\mathbf{f}$ comparing adults with children 
Table 2 Risk of progression to active TB across IFN-gamma levels (IU/ml) by risk group

\begin{tabular}{|c|c|c|c|c|}
\hline \multirow{2}{*}{$\begin{array}{l}\text { IFN-gamma } \\
\text { levels (IU/ml) }\end{array}$} & \multicolumn{4}{|c|}{ Relative Risk (95\% Uncertainty interval) } \\
\hline & People living with HIV & TB Case Contacts & Healthcare workers & Migrants \\
\hline 0.00 & REF & REF & REF & REF \\
\hline 0.10 & 1.28 (1.00 to 2.33$)$ & $1.26(1.12$ to 1.40$)$ & $1.20(1.00$ to 1.79$)$ & 1.25 (1.11 to 1.42$)$ \\
\hline 0.20 & 1.51 (1.00 to 3.62$)$ & 1.51 (1.24 to 1.78$)$ & 1.37 (1.00 to 2.57$)$ & 1.50 (1.22 to 1.84$)$ \\
\hline 0.30 & $1.73(1.00$ to 4.88$)$ & 1.76 (1.37 to 2.16$)$ & 1.54 (1.00 to 3.35$)$ & 1.73 (1.33 to 2.24$)$ \\
\hline 0.35 & $1.83(1.00$ to 5.50$)$ & 1.88 (1.43 to 2.34$)$ & $1.62(1.00$ to 3.74$)$ & 1.85 (1.39 to 2.43$)$ \\
\hline 0.40 & 1.94 (1.01 to 6.11$)$ & 2.00 (1.49 to 2.52) & $1.70(1.01$ to 4.12$)$ & 1.96 (1.44 to 2.62) \\
\hline 0.50 & $2.14(1.01$ to 7.36$)$ & 2.23 (1.62 to 2.87$)$ & $1.85(1.01$ to 4.89$)$ & 2.19 (1.56 to 3.00$)$ \\
\hline 0.70 & 2.56 (1.01 to 9.85$)$ & 2.69 (1.89 to 3.54) & $2.16(1.02$ to 6.39$)$ & $2.62(1.78$ to 3.71$)$ \\
\hline 1.00 & $3.21(1.02$ to 13.27$)$ & 3.33 (2.29 to 4.46$)$ & 2.61 (1.02 to 8.34$)$ & $3.23(2.12$ to 4.68$)$ \\
\hline 2.00 & 5.62 (1.10 to 24.32$)$ & 5.17 (3.62 to 6.92) & 4.15 (1.05 to 14.45$)$ & 4.93 (3.32 to 7.27 ) \\
\hline 3.00 & 8.39 (1.35 to 34.57$)$ & 6.58 (4.61 to 8.77$)$ & 5.69 (1.16 to 19.84$)$ & 6.16 (4.18 to 8.95$)$ \\
\hline 4.00 & 11.50 (1.73 to 45.48$)$ & 7.62 (5.01 to 10.29$)$ & 7.24 (1.33 to 26.21$)$ & $7.02(4.43$ to 10.26$)$ \\
\hline 5.00 & 14.99 (2.49 to 55.66$)$ & 8.39 (5.28 to 11.66$)$ & 8.84 (1.65 to 32.72) & 7.61 (4.53 to 11.10$)$ \\
\hline 6.00 & $18.83(3.56$ to 67.91$)$ & 9.01 (5.47 to 13.16$)$ & 10.53 (2.09 to 38.31$)$ & 8.06 (4.62 to 12.33$)$ \\
\hline 7.00 & 23.00 (5.07 to 78.98$)$ & 9.60 (5.95 to 14.44$)$ & 12.35 (2.75 to 43.89$)$ & 8.49 (4.96 to 13.43$)$ \\
\hline 8.00 & 27.30 (6.68 to 94.05$)$ & $10.21(6.77$ to 15.17$)$ & 14.26 (3.56 to 48.25$)$ & 8.97 (5.58 to 14.43 ) \\
\hline 10.00 & 35.34 (9.59 to 119.43 ) & 11.37 (7.98 to 16.53$)$ & 17.84 (5.06 to 60.08) & 9.94 (6.44 to 15.71$)$ \\
\hline 12.00 & 41.74 (11.25 to 146.63$)$ & $12.33(8.49$ to 18.17$)$ & 20.73 (5.87 to 72.65 ) & 10.79 (6.81 to 17.48$)$ \\
\hline 15.00 & 46.86 (13.17 to 173.69$)$ & 13.35 (9.01 to 20.46) & 23.20 (6.66 to 82.24$)$ & 11.72 (7.17 to 19.42$)$ \\
\hline 20.00 & $47.70(13.46$ to 175.51$)$ & 13.84 (9.24 to 21.09) & 23.85 (7.02 to 83.46$)$ & $12.19(7.57$ to 20.11$)$ \\
\hline
\end{tabular}

to the quality of the studies as the results did not differ significantly by the quality of studies.

We found that the risk of TB is higher with larger IFN-gamma levels. Our findings show that the risk for incident $\mathrm{TB}$ is 2.90 -fold higher at $1 \mathrm{IU} / \mathrm{ml}, 10.38$-fold higher at $5 \mathrm{IU} / \mathrm{ml}, 19.00$-fold higher at $10 \mathrm{IU} / \mathrm{ml}, 21.82$ fold higher at $15 \mathrm{IU} / \mathrm{ml}$, and 22.31-fold higher at $20 \mathrm{IU} /$ $\mathrm{ml}$. These findings underscore a limitation of the current practice of dichotomizing IGRA results where the interpretation is that risk is constant for all positive IGRA test. However, our findings show that the risk of active TB development is not the same for everyone with a positive IGRA when considering the full spectrum of positive IGRA reactions. In addition, the results indicate that very high IFN-gamma levels from a QuantiFERONTB test, beyond the traditional threshold, are powerful indicators of progression to active TB from latent infection. Some authors have suggested that these very high levels of IFN-gamma levels are markers for subclinical active TB disease or early incipient disease [12, 18].

The dose-response relationship found in this metaanalysis can be used to help guide clinical decisions to perform additional tests and treat latent tuberculosis infection within the context of TB programs and local epidemiology. Guidance in clinical decisions would particularly be useful in high $\mathrm{TB}$ burden areas where preventive treatment for latent TB is under-utilized [19, 20]. The results from this meta-analysis can help make clinical decisions more efficient when results are in the intermediate area between a negative and positive IGRA test called the borderline zone. A recent large cohort study found that half of the patients with an IFN-gamma level in the borderline zone $(0.20-1.00 \mathrm{IU} / \mathrm{ml})$ were IGRA negative in a follow-up test [14]. Results in this borderline zone can provide justification for secondary IGRA tests to prevent unnecessary treatment. Furthermore, our results show that the risk of incident TB is markedly greater in people with high IFN-gamma levels. Current WHO recommendations are to provide preventive treatment to a subset of risk groups such as PLHIV and TB case contacts [8]. The findings from this study suggest an opportunity to consider the potential benefit of extending TB preventive treatment to those with high IFN-gamma levels while taking into account risk factors for disease progression.

We found some variation in the dose-response relationship by population. Compared to our primary curve that excluded studies where the entire study population is HIV positive, the dose-response relationship was substantially higher in studies among HIV positive individuals. Though some studies have found that IGRAs have modest predictive power for incident TB among HIV 
positive individuals [21, 22], our results are consistent with studies indicating that IGRAs are sensitive tools for predicting TB progression among PLHIV [23-25].

In addition, we found some differences in the doseresponse relationship when stratifying analyses by adults and children. The relative risk of incident TB was lower for children compared to adults at the lower end of the curve before converging at $7.5 \mathrm{IU} / \mathrm{ml}$. The different risk is likely due to mixed findings on the utility of IGRA's among very young children. Several studies have found that there is insufficient reaction to $M$. tuberculosis antigen among young children that adversely impacts IGRA results [26-28]. More investigations are needed to assess the efficacy of IGRAs to predict progression to active TB to confirm the performance of IGRA at lower levels of IFN-gamma levels.

Contrary to expectations, we found no evidence of effect modification when stratifying models by whether studies provided preventive treatment for TB. The lack of difference in the curves can be due to the fact that only a small proportion of participants accepted treatment in studies where it was provided. In addition, even a smaller fraction of those accepting treatment completed the regimen in these studies.

Finally, the findings from this study bring into question the predictive value for progression to active TB of IGRAs. Various systematic reviews have concluded that IGRAs have poor accuracy for the prediction of incident TB [21, 29]. These results may change by taking into account the full dynamic nature of latent infection instead of dichotomizing IGRA results with conventional thresholds. In studies where IFN-gamma levels were evaluated at larger cutoffs beside the threshold of $0.35 \mathrm{IU} / \mathrm{ml}$, the predictive value of subsequent TB improved [10, 12, 30, 31].

\section{Strengths and limitations}

Our study has several key strengths. In our metaregression we were able to include data with different IFN-gamma categories into a singular analysis while incorporating between study heterogeneity in our uncertainty estimation. This is an advantage over traditional methods that would have to take the midpoint of IFN-gamma categories rather than use information of the entire category as done in this study. Our study is the first meta-analysis to examine TB risk over the entire distribution of IFN-gamma levels, allowing for improved identification of individuals who may be at highest risk for progressing to active TB. Finally, we were able to stratify results by important at risk populations to evaluate for potential confounding and effect modification.

Our findings should be interpreted in terms of key limitations. First, we could not assess for effect modification by known risk factors for progression to active TB including tobacco smoke, alcohol consumption, diabetes, malnutrition, and indoor air pollution, as these data were not routinely included in studies. Second, several studies included in our systematic review used passivefollow-up for detection of active TB through national TB registries. These surveillance systems are often prone to under-reporting which may have caused lower rates of observed TB cases in the studies. Third, our quality assessment indicated that almost all studies have some source of bias as all studies were considered to be of low to moderate quality. We conducted sensitivity analyses to evaluate the impact of study quality on the results, and we found that the results did not differ significantly. Fourth, most studies were conducted in low to intermediate TB burden countries, potentially limiting the generalizability of our findings. Fifth, time since infection may impact results as recent infection is associated with higher risk for active TB. However, this information was unavailable in most studies. Finally, we may have missed articles as we restricted our search to two databases. We believe this had a small impact on our findings because PubMed and Embase yield high coverage [32] and we manually searched the reference list of relevant articles.

Our study has implications for future studies. We found that the risk of incident TB is not the same for everyone with a positive IGRA reaction. Future cohort studies should therefore collect granular data on IGRA levels and the associated risk of progression to active tuberculosis. For example, out of the 34 included studies in our systematic review, only 9 reported more IGRA values besides the traditional cutoff of $0.35 \mathrm{IU} / \mathrm{ml}$. Reporting more granular IGRA values and corresponding risks for progression to active $\mathrm{TB}$ will also help reassess the predictive value of IGRAs given the doseresponse nature of the data. Future studies may also incorporate additional risk factors for progression to active tuberculosis such as alcohol, smoking, malnutrition, and diabetes to identify individuals at greatest risk of subsequent TB.

\section{Conclusion}

We developed a dose-response risk curve for the progression to active $\mathrm{TB}$ as a function of a continuous measure of IGRA values. Our findings show that the current practice of dichotomizing IGRA test results simplifies the TB infection disease continuum. The findings of this study showed that the risk of active TB development is not the same for everyone with a positive IGRA, with higher IGRA values being strongly associated with disease progression. With IGRAs starting to scale up in high TB burden areas, the findings from this study can help clinicians make informed decisions by providing different relative risks of progression to active TB for a range of IGRA values within the borderline zone and 
very high IGRA reactions. More investigations using detailed quantification of IGRA values will help to find more accurate estimates of the dose-response relationship and allow for a reexamination of the predictive power of IGRA tests.

\section{Abbreviations}

TB: Tuberculosis; IGRA: Interferon-gamma release assay; PRISMA: Preferred Reporting Items for Systematic Review; MOOSE: Meta-analysis of Observational Studies in Epidemiology; LTBI: Latent Tuberculosis Infection; TST: Tuberculin Skin Test; QFT-GIT: QuantiFERON TB Gold in Tube; WHO: World Health Organization; NOS: Newcastle-Ottawa Quality Assessment Scale; PLHIV: People living with HIV; TPT: Tuberculosis Preventive Treatment

\section{Supplementary Information}

The online version contains supplementary material available at https://doi. org/10.1186/s12879-021-06141-4.

Additional file 1: Supplementary file S1: Data Supplement. Table S1 Characteristics of the studies included in the meta-analysis. Table S2. Search strategies. Table S3. Newcastle-Ottawa quality assessment scale adopted for quality assessment. Figure S1. Sensitivity analysis results (comparing time to incident TB of 2 or 3 months to 6 months or more). Figure S2. Sensitivity analysis results (comparing bacteriologically confirmed TB to clinically confirmed TB). Figure S3. Sensitivity analysis results (comparing all form TB incidence in country of study by $<40$ per 100,000 and $>40$ per 100,000). Figure S4. Sensitivity analysis results (excluding studies that provided TB preventive treatment). PRISMA-P 2015 Checklist.

\section{Acknowledgements}

Not applicable.

\section{Authors' contributions}

Study concept and design: JRL, HHK, TV, JMR, PZ. Development and implementation of literature search strategy: JRL, HHK, JM. Acquisition of data, including review of literature search results and data abstraction: JRL, HHK, JMR, JM. Analysis and interpretation of data: JRL, HHK, TV, JMR, PZ. Drafting of the manuscript: JRL, HHK, TV, JMR, PZ. Critical revision of the manuscript for important intellectual content: all authors. Statistical analysis: JRL, JM, PZ. Study supervision: HHK. All authors have read and approved the final manuscript.

\section{Funding}

Bill \& Melinda Gates Foundation. The funder had no role in study design, data collection, data analysis, data interpretation, or the writing of the report.

\section{Availability of data and materials}

The data sets used and/or analyzed during the current study are available from the corresponding author on reasonable request.

\section{Declarations}

\section{Ethical approval and consent to participate}

Not applicable.

\section{Consent for publication}

Not applicable.

\section{Competing interests}

The authors declare that they have no competing interests.

\section{Author details}

${ }^{1}$ Institute for Health Metrics and Evaluation, University of Washington, 3980 15th Ave. NE, Seattle, WA 98195, USA. ²Department of Health Metrics Sciences, University of Washington, 3980 15th Ave. NE, Seattle, WA 98195, USA. ${ }^{3}$ Department of Global Health, University of Washington, 325 9th Avenue, Box 359931, Seattle, WA 98104, USA. ${ }^{4}$ Department of Medicine,
University of Washington, 1959 NE Pacific Street, Box 356420, Seattle, WA 98195, USA.

Received: 1 December 2020 Accepted: 5 May 2021

Published online: 22 May 2021

\section{References}

1. Vos T, Lim SS, Abbafati C, Abbas KM, Abbasi M, Abbasifard M, et al. Global burden of 369 diseases and injuries in 204 countries and territories, 19902019: a systematic analysis for the global burden of disease study 2019. Lancet. 2020;396(10258):1204-22 Available from: https://linkinghub.elsevier com/retrieve/pii/S0140673620309259.

2. James SL, Abate D, Abate KH, Abay SM, Abbafati C, Abbasi N, et al. Global, regional, and national incidence, prevalence, and years lived with disability for 354 diseases and injuries for 195 countries and territories, 1990-2017: a systematic analysis for the global burden of disease study 2017. Lancet. 2018;392(10159):1789-858 Available from: https://linkinghub.elsevier.com/ retrieve/pii/S0140673618323353.

3. Houben RMGJ, Dodd PJ. The global burden of latent tuberculosis infection: a re-estimation using mathematical Modelling. PLoS Med. 2016;13(10): e1002152 Available from: http://www.ncbi.nlm.nih.gov/pubmed/27780211.

4. Kiazyk S, Ball TB. Latent tuberculosis infection: an overview. Can Commun Dis Rep. 2017;43(3-4):62-6 Available from: http://www.ncbi.nlm.nih.gov/ pubmed/29770066.

5. Rangaka MX, Cavalcante SC, Marais BJ, Thim S, Martinson NA, Swaminathan $S$, et al. Controlling the seedbeds of tuberculosis: diagnosis and treatment of tuberculosis infection. Lancet. 2015;386(10010):2344-53 Available from: https://linkinghub.elsevier.com/retrieve/pii/S0140673615003232.

6. Gualano G, Mencarini P, Lauria FN, Palmieri F, Mfinanga S, Mwaba P, et al. Tuberculin skin test - outdated or still useful for latent TB infection screening? Int J Infect Dis. 2019;80:S20-2 Available from: https://inkinghub. elsevier.com/retrieve/pii/S120197121930061X.

7. Trajman A, Steffen RE, Menzies D. Interferon-gamma release assays versus tuberculin skin testing for the diagnosis of latent tuberculosis infection: an overview of the evidence. Pulm Med. 2013;2013:1-11 Available from: http:// www.hindawi.com/journals/pm/2013/601737/.

8. World Health Organization (WHO). Global tuberculosis report 2019. https://a pps.who.int/iris/bitstream/handle/10665/329368/9789241565714-eng. pdf?ua=1. Accessed 23 June 2020.

9. Campbell JR, Winters N, Menzies D. Absolute risk of tuberculosis among untreated populations with a positive tuberculin skin test or interferongamma release assay result: systematic review and meta-analysis. BMJ. 2020; m549 Available from: http://www.bmj.com/lookup/doi/10.1136/bmj.m549.

10. Winje BA, White $R$, Syre H, Skutlaberg DH, Oftung F, Mengshoel AT, et al. Stratification by interferon- $\gamma$ release assay level predicts risk of incident TB. Thorax. 2018;73(7):652-61 Available from: http://thorax.bmj.com/lookup/ doi/10.1136/thoraxjnl-2017-211147.

11. Zellweger J-P, Sotgiu G, Block M, Dore S, Altet N, Blunschi R, et al. Risk assessment of tuberculosis in contacts by IFN- $\gamma$ release assays. A tuberculosis network European trials group study. Am J Respir Crit Care Med. 2015;191(10):1176-84. https://doi.org/10.1164/rccm.201502-0232OC.

12. Andrews JR, Nemes E, Tameris M, Landry BS, Mahomed H, McClain JB, et al. Serial QuantiFERON testing and tuberculosis disease risk among young children: an observational cohort study. Lancet Respir Med. 2017;5(4):282-90 Available from: https://linkinghub.elsevier.com/retrieve/pii/S22132600173 00607.

13. Hermansen TS, Lillebaek T, Langholz Kristensen $\mathrm{K}$, Andersen PH, Ravn P. Prognostic value of interferon- $\gamma$ release assays, a population-based study from a TB low-incidence country. Thorax. 2016;71(7):652-8 Available from: http://thorax.bmj.com/lookup/doi/10.1136/thoraxjnl-2015-208228.

14. Jonsson J, Westman A, Bruchfeld J, Sturegård E, Gaines H, Schön T. A borderline range for Quantiferon gold in-tube results. Shams (Amir) Homayoun, editor. PLoS One. 2017;12(11):e0187313. https://doi.org/10.1371/ journal.pone.0187313.

15. Nienhaus A, Costa JT. Screening for tuberculosis and the use of a borderline zone for the interpretation of the interferon- $\gamma$ release assay (IGRA) in Portuguese healthcare workers. J Occup Med Toxicol. 2013;8(1):1 Available from: http://occup-med.biomedcentral.com/articles/10.1186/1745-6673-8-1.

16. Wells G, Shea B, O'Connell D, Peterson J, Welch V, Losos M, et al. The Newcastle-Ottawa scale (NOS) for assessing the quality of nonrandomised studies in meta-analyses: Department of Epidemiology and Community 
Medicine, University of Ottawa, Canada; 2011. Available from: http://www. ohri.ca/programs/clinical_epidemiology/oxford.asp

17. Zheng P, Aravkin AY, Barber R, Sorensen RJD, Murray CJL. Trimmed constrained mixed effects models: formulations and algorithms; 2019. Available from: http://arxiv.org/abs/1909.10700

18. Drain PK, Bajema KL, Dowdy D, Dheda K, Naidoo K, Schumacher SG, et al. Incipient and subclinical tuberculosis: a clinical review of early stages and progression of infection. Clin Microbiol Rev. 2018;31(4) Available from: http://www.ncbi.nlm.nih.gov/pubmed/30021818.

19. Churchyard GJ, Swindells S. Controlling latent TB tuberculosis infection in high-burden countries: a neglected strategy to end TB. PLoS Med. 2019; 16(4):e1002787 Available from: http://www.ncbi.nlm.nih.gov/pubmed/31 013273

20. Sharma SK, Mohanan S, Sharma A. Relevance of latent TB infection in areas of high TB prevalence. Chest. 2012;142(3):761-73 Available from: https:// linkinghub.elsevier.com/retrieve/pii/S0012369212605224.

21. Cattamanchi A, Smith R, Steingart KR, Metcalfe JZ, Date A, Coleman C, et al. Interferon-gamma release assays for the diagnosis of latent tuberculosis infection in HIV-infected individuals: a systematic review and meta-analysis. JAIDS J Acquir Immune Defic Syndr. 2011;56(3):230-8 Available from: http:// content.wkhealth.com/linkback/openurl?sid=WKPTLP:landingpage\&an $=0012$ 6334-201103010-00006

22. Sester M, van Leth F, Bruchfeld J, Bumbacea D, Cirillo DM, Dilektasli AG, et al. Risk assessment of tuberculosis in Immunocompromised patients. A TBNET study. Am J Respir Crit Care Med. 2014;190(10):1168-76. https://doi. org/10.1164/rccm.201405-09670C

23. Aichelburg MC, Rieger A, Breitenecker F, Pfistershammer K, Tittes J, Eltz S, et al. Detection and prediction of active tuberculosis disease by a wholeblood interferon- $\gamma$ release assay in HIV-1-infected individuals. Clin Infect Dis. 2009;48(7):954-62 Available from: https://academic.oup.com/cid/articlelookup/doi/10.1086/597351.

24. Doyle JS, Bissessor M, Denholm JT, Ryan N, Fairley CK, Leslie DE. Latent tuberculosis screening using interferon-gamma release assays in an Australian HIV-infected cohort. JAIDS J Acquir Immune Defic Syndr. 2014; 66(1):48-54 Available from: http://content.wkhealth.com/linkback/ openurl?sid=WKPTLP:landingpage\&an=00126334-201405010-00007.

25. Lee S, Lee JE, Kang JS, Lee SO, Lee SH. Long-term performance of the IGRA to predict and prevent active tuberculosis development in HIV-infected patients. Int J Tuberc Lung Dis. 2019;23(4):422-7 Available from: https:// www.ingentaconnect.com/content/10.5588/ijtld.18.0198.

26. Lewinsohn DA, Lobato MN, Jereb JA. Interferon- $\gamma$ release assays: new diagnostic tests for mycobacterium tuberculosis infection, and their use in children. Curr Opin Pediatr. 2010;22(1):71-6 Available from: http://journals. Iww.com/00008480-201002000-00013.

27. Haustein T, Ridout DA, Hartley JC, Thaker U, Shingadia D, Klein NJ, et al. The likelihood of an indeterminate test result from a whole-blood interferon- $\gamma$ release assay for the diagnosis of mycobacterium tuberculosis infection in children correlates with age and immune status. Pediatr Infect Dis J. 2009; 28(8):669-73 Available from: http://journals.Iww.com/00006454-20090800000001.

28. Lighter J, Rigaud M, Eduardo R, Peng C-H, Pollack H. Latent tuberculosis diagnosis in children by using the QuantiFERON-TB gold in-tube test. Pediatrics. 2009:123(1):30-7. https://doi.org/10.1542/peds.2007-3618.

29. Rangaka MX, Wilkinson KA, Glynn JR, Ling D, Menzies D, MwansaKambafwile J, et al. Predictive value of interferon- $\gamma$ release assays for incident active tuberculosis: a systematic review and meta-analysis. Lancet Infect Dis. 2012;12(1):45-55 Available from: https://linkinghub.elsevier.com/ retrieve/pii/S1473309911702109.

30. Altet N, Dominguez J, de Souza-Galvão M-L, Jiménez-Fuentes MÁ, Milà C, Solsona J, et al. Predicting the development of tuberculosis with the tuberculin skin test and QuantiFERON testing. Ann Am Thorac Soc. 2015; 12(5):680-8. https://doi.org/10.1513/AnnalsATS.201408-394OC
31. Gupta RK, Lipman M, Jackson C, Sitch AJ, Southern J, Drobniewski F, et al. Quantitative IFN- $\gamma$ release assay and tuberculin skin test results to predict incident tuberculosis. A prospective cohort study. Am J Respir Crit Care Med. 2020;201 (8):984-91. https://doi.org/10.1164/rccm.201905-09690C.

32. Bramer WM, Giustini D, Kramer BMR. Comparing the coverage, recall, and precision of searches for 120 systematic reviews in Embase, MEDLINE, and Google scholar: a prospective study. Syst Rev. 2016;5(1):39 Available from: http://www.systematicreviewsjournal.com/content/5/1/39.

\section{Publisher's Note}

Springer Nature remains neutral with regard to jurisdictional claims in published maps and institutional affiliations.

\section{Ready to submit your research? Choose BMC and benefit from:}

- fast, convenient online submission

- thorough peer review by experienced researchers in your field

- rapid publication on acceptance

- support for research data, including large and complex data types

- gold Open Access which fosters wider collaboration and increased citations

- maximum visibility for your research: over $100 \mathrm{M}$ website views per year

At BMC, research is always in progress.

Learn more biomedcentral.com/submissions 